\title{
INTERACTION OF SYMBOLIC STATES IN ATOMIC STRUCTURE COMPUTATIONS
}

\author{
R. Matulioniené ${ }^{\mathrm{a}}$, D. Ellis ${ }^{\mathrm{a}}$, and C. Froese Fischer ${ }^{\mathrm{b}}$ \\ ${ }^{a}$ Department of Physics \& Astronomy MS 111, The University of Toledo, Toledo, Ohio 43606, USA \\ E-mail: dellis@utnet.utoledo.edu \\ ${ }^{\mathrm{b}}$ Department of Computer Science, Box 1679B, Vanderbilt University, Nashville, Tennessee 37235, USA
}

Received 15 October 2007; accepted 21 November 2007

\begin{abstract}
We derive general equations for angular coefficients needed to carry out atomic structure computations using symbolic state expansions. In this new approach the energy is expressed, not in terms of kinetic energy and Slater integrals, but in terms of two-electron matrix elements, with coefficients that are independent of the one-electron quantum numbers involved in these matrix elements. Specific results are given for the matrix elements of a symmetric scalar two-body operator involving singlereplacement and double-replacement symbolic states. The derivations use $j j$ coupling, coefficients of fractional parentage for nonequivalent electrons, and diagrammatic angular momentum algebra.
\end{abstract}

Keywords: atomic structure theory, symbolic state expansion, angular momentum algebra

PACS: $31.15 .-p$

\section{Introduction}

Large scale atomic structure computations require the repeated evaluation of many interaction matrix elements, each of which is the product of a radial and an angular integral. The results of the angular integrations do not change as the radial functions are adjusted during the course of a calculation, so they are typically computed at the beginning and stored. However the quantity of this angular data grows rapidly with the size of the computation. In the effort to develop computer programs capable of producing accurate atomic data, the storage and retrieval of angular data is becoming a serious issue. One method for addressing this problem is the symbolic state expansion, proposed by Froese Fischer [1]. In this approach, a symbolic state is defined as a configuration state in which the quantum numbers of the electron orbitals are not fully specified. The number of basis states used can then be increased systematically during the course of a calculation without changing the number of symbolic states. The angular parts of the integrations for the required matrix elements between symbolic states can be worked out at the beginning and saved. Then the size of the calculation can be increased without recalculating this angular data.
In this paper we derive the angular coefficients needed for the matrix elements of a two-body operator in this new scheme. Because of the number of angular momenta which must be recoupled and the complications of anti-symmetry, the derivations depend on two concepts in addition to standard angular-momentum algebra: the diagrammatic method of Jucys [2] and the coefficients of fractional parentage for nonequivalent electrons (MCFP) of Meshkov [3] and Armstrong [4, 5]. There are a number of different graphical methods of performing angular integrals [6-9], most growing from the original work of Jucys [10]. In this paper we specifically use the scheme presented by Jucys and Bandzaitis [2]; following Rudzikas [11] we refer to these as JB graphs. All the results of this paper have been checked in detail by the use of ordinary angular-momentum algebra, following Rotenberg et al. [12]. Because of the pressing need for relativistic calculations in heavy atoms and ions, we do the derivations in $j j$ coupling. The corresponding results for LS coupling have been worked out in a similar way [13] and are given in reference [1]. 


\section{The symbolic expansion}

Let us consider an atom or ion with $N$ electrons; the multiconfiguration expansion of a general atomic state can be written in terms of symbolic states:

$\left|\Psi_{\text {atom }}\right\rangle=\sum_{\text {ref }} c_{\text {ref }}\left|\Psi_{\text {ref }}\right\rangle+\sum_{d} c_{d}\left|\Psi_{d}\right\rangle+\sum_{s} c_{s}\left|\Psi_{s}\right\rangle$.

Here, $\left|\Psi_{\text {ref }}\right\rangle$ is a configuration state function belonging to the reference set. The ket $\left|\Psi_{d}\right\rangle$ represents a symbolic state formed by a two-electron replacement from the reference set - a double-replacement symbolic state, and $\left|\Psi_{s}\right\rangle$ is a single-replacement symbolic state obtained in a similar way:

$$
\begin{aligned}
& \left|\Psi_{d}\right\rangle=\left|\left\{\left(\Psi_{\text {sub }}\right) J_{\text {sub }},\left(n_{1} l_{1} j_{1}, n_{2} l_{2} j_{2}\right) J_{v}\right\} J_{\text {tot }}\right\rangle, \\
& \left|\Psi_{s}\right\rangle=\left|\left\{\left(\Psi_{\text {sub }}\right) J_{\text {sub }},\left(n_{v} l_{v} j_{v}\right)\right\} J_{\text {tot }}\right\rangle .
\end{aligned}
$$

In the double-replacement symbolic state, $\Psi_{\text {sub }}$ represents a subconfiguration: that is, a coupled antisymmmetric state of $N-2$ electrons, formed by removing two orbitals from a reference state. Likewise $\left(n_{1} l_{1} j_{1}, n_{2} l_{2} j_{2}\right) J_{v}$ is a coupled antisymmetric 2-electron state formed from a pair of correlation orbitals. Here we assume that all these correlation orbitals are individually orthogonal to all orbitals used in the reference set. Finally the $(N-2)$-electron subconfiguration and the 2-electron coupled pair are coupled together to form the fully antisymmetric $N$-electron state function $\left|\Psi_{d}\right\rangle$. Having thus defined $\left|\Psi_{d}\right\rangle$, the structure of $\left|\Psi_{s}\right\rangle$ is self-explanatory. The crucial point is that, whereas the reference set is given, and $\Psi_{\text {sub }}, J_{v}, J_{\text {tot }}$ are specified, the $n l j$ quantum numbers of the correlation orbitals remain unspecified.

One way to generate an expansion such as (1) is to expand one or more of the reference functions by "pulling out" one or two electrons in all possible ways:

$$
\begin{aligned}
& \left|\Psi_{\text {ref }}(1, \ldots, N) J_{\text {tot }}\right\rangle= \\
& \left.\sum_{\substack{\left(\Psi_{\text {sub }}\right) J_{\text {sub }},\left(\psi_{p}\right) J_{v}}}\left(\Psi_{\text {ref }} \mid\right\} \Psi_{\text {sub }}, \psi_{p}\right) \\
& \left|\left\{\Psi_{\text {sub }}(1, \ldots, N-2) J_{\text {sub }}, \psi_{p}(N-1, N) J_{v}\right\} J_{\text {tot }}\right\rangle .
\end{aligned}
$$

Here, $\left.\left(\Psi_{\text {ref }} \mid\right\} \Psi_{\text {sub }}, \psi_{p}\right)$ is a coefficient of fractional parentage for nonequivalent electrons (MCFP). Note that while $\Psi_{\text {ref }}, \Psi_{\text {sub }}$, and $\psi_{p}$ are fully antisymmetric, the newly formed $\mathrm{N}$-electron states are not individually antisymmetric, just as in the usual fractional parentage expansion. Now each term in (4) can be used to form a symbolic state $\left|\Psi_{d}\right\rangle$ by first replacing both the orbitals in the "pullout" state function $\psi_{p}(N-1, N)$ by correlation orbitals that are unoccupied in all the reference state functions, and then summing over electron label permutations to form a fully antisymmetric $N$-electron state function $\left|\Psi_{d}\right\rangle$. Obviously $\left|\Psi_{s}\right\rangle$ can be formed analogously.

Using the standard basis of configuration state functions, a general matrix element is expressed as a product of an angular factor and a radial factor. By contrast, in the symbolic state method there will be a sum of products of three factors: (1) an angular factor independent of the correlation orbitals, (2) an angular factor depending on the quantum numbers of the correlation orbitals, (3) a two-electron matrix element, containing both angular and radial factors [1]. It is our purpose in the present work to derive explicit expressions for these angular factors in the case of a two-body operator, which will be needed in the construction of Hamiltonian matrix elements.

\section{Two-electron matrix elements}

We consider a symmetric scalar two-body operator $\mathbf{G}=\sum G_{i j}$, where $G_{i j}=G_{j i}$ acts on electrons $i, j$ and commutes with the total angular momentum operator, and the sum is over all electron pairs, $1 \leq i<j \leq N$. The general form of such an operator is

$$
G_{i j}=\sum_{g}\left(G^{(g)}(i) \cdot G^{(g)}(j)\right) \mathcal{R}_{g}(i, j),
$$

where $G^{(g)}$ is a one-electron spherical tensor operator of rank $g$ and $\mathcal{R}_{g}$ is a scalar. We want to express a matrix element of $\mathbf{G}$ between the described $N$-electron states in terms of matrix elements between coupled antisymmetric two-electron states:

$$
\begin{aligned}
\left\langle\Psi^{\prime}|\mathbf{G}| \Psi\right\rangle= & \sum_{\alpha, \beta} K_{\alpha \beta}\left(\Psi^{\prime}, \Psi\right) \\
& \times\left\langle\psi_{\alpha}^{\prime}(1,2)\left|G_{12}\right| \psi_{\beta}(1,2)\right\rangle .
\end{aligned}
$$

Here $\left|\psi_{\alpha}(1,2)\right\rangle$ is an antisymmetric coupled state of electrons 1 and 2, and we label all such states by the indices $\alpha, \beta$. This expansion is symbolic, since the quantum numbers of the correlation orbitals are not specified. We want to determine which two-electron states $\psi_{\alpha}^{\prime}, \psi_{\beta}$ occur in this expansion, and to study the dependence of the coefficients $K_{\alpha \beta}$ on the quantum numbers of the correlation orbitals. 
In a calculation based on (1) we will need matrix elements of form $\left\langle\Psi_{\text {atom }}^{\prime}|\mathbf{G}| \Psi_{\text {atom }}\right\rangle$, where $\mathbf{G}$ is the twoelectron part of the hamiltonian operator. Matrix elements of type $\left\langle\Psi_{\text {ref }}^{\prime}|\mathbf{G}| \Psi_{\text {ref }}\right\rangle$ can be expanded in terms of two-electron matrix elements by use of a standard textbook approach [14]. In the sections that follow, we focus on the five distinct types of matrix elements involving symbolic states:

$$
\begin{gathered}
\left\langle\Psi_{d}|\mathbf{G}| \Psi_{\mathrm{ref}}\right\rangle,\left\langle\Psi_{s}|\mathbf{G}| \Psi_{\mathrm{ref}}\right\rangle,\left\langle\Psi_{d}^{\prime}|\mathbf{G}| \Psi_{d}\right\rangle, \\
\left\langle\Psi_{s}^{\prime}|\mathbf{G}| \Psi_{s}\right\rangle,\left\langle\Psi_{s}^{\prime}|\mathbf{G}| \Psi_{d}\right\rangle .
\end{gathered}
$$

Here the state of each electron is described by a 4-component Dirac spinor, the number of electrons is arbitrary, and the states in the reference set contain any number of closed and open shells. Graphical methods of angular momentum calculation offer a convenient way to tackle the coupling details of the problem in all generality. However, many-electron states are not only coupled, but also antisymmetric under interchange on any two electron coordinates. To take advantage of graphical angular momentum techniques, we have to attack antisymmetrization complexities first.

\section{Antisymmetrization issues}

We find that antisymmetrization details simplify significantly if the required matrix elements are expressed in terms of coupled but not fully antisymmetrized functions in which the correlation orbitals and the subconfiguration are separately antisymmetric. To disentangle the correlation orbitals from the subconfiguration in the doublereplacement symbolic states, we make the following definitions:

$$
\begin{array}{r}
\left|\Phi_{d}\right\rangle \equiv\left|\left\{\Psi_{\text {sub }}(1,2 \cdots N-2) J_{\text {sub }}, \psi_{v}(N-1, N) J_{v}\right\} J_{\text {tot }}\right\rangle, \\
\left|\Phi_{d}^{X}\right\rangle \equiv\left|\left\{\Psi_{\text {sub }}(1,2 \cdots N-3, N-1) J_{\text {sub }}, \psi_{v}(N-2, N) J_{v}\right\} J_{\text {tot }}\right\rangle .
\end{array}
$$

Here, $\psi_{v}(N-1, N)$ is a normalized, coupled, and antisymmetric state of the correlation pair with electron labels $N-1, N$, and $\Psi_{\text {sub }}(1,2 \cdots N-2)$ is a normalized, coupled, and antisymmetric subconfiguration of electrons $1,2, \ldots, N-2$. The state $\left|\Phi_{d}^{X}\right\rangle$ is obtained from $\left|\Phi_{d}\right\rangle$ by interchanging electron labels $N-2$ and $N-1$. The states $\left|\Phi_{d}\right\rangle$ and $\left|\Phi_{d}^{X}\right\rangle$ are obviously not fully antisymmetric.

The corresponding definitions for single-replacement symbolic states are

$$
\begin{aligned}
\left|\Phi_{s}\right\rangle & \equiv\left|\left\{\Psi_{\text {sub }}(1,2 \cdots N-1) J_{\text {sub }}, \psi_{v}(N) j_{v}\right\} J_{\text {tot }}\right\rangle, \\
\left|\Phi_{s}^{X}\right\rangle & \equiv\left|\left\{\Psi_{\text {sub }}(1,2 \cdots N-2, N) J_{\text {sub }}, \psi_{v}(N-1) j_{v}\right\} J_{\text {tot }}\right\rangle .
\end{aligned}
$$

The fully antisymmetric states $\left|\Psi_{d}\right\rangle,\left|\Psi_{s}\right\rangle$ can be expressed in terms of partially antisymmetrized states like $\left|\Phi_{d}\right\rangle$, $\left|\Phi_{s}\right\rangle$ by summing over permutations of electron labels. Since $\psi_{v}$ and $\Psi_{\text {sub }}$ are already antisymmetric individually, we include only those permutations by which electron labels are moved from $\psi_{v}$ to $\Psi_{\text {sub }}$ and vice versa. Within both $\psi_{v}$ and $\Psi_{\text {sub }}$ the label ordering remains standard, with increasing numerical value of the electron labels.

Let us first consider a double-replacement symbolic state. We specify a permutation by an interchange of electron labels $N-1, N$ with an arbitrary pair of $\Psi_{\text {sub }}$ electron labels $a, b$. Then, by counting the number of label interchanges required to get back to the original ordering, $\Psi_{\text {sub }}(1,2 \cdots N-2) \psi_{v}(N-1, N)$, we can show that the sign of this permutation is $(-1)^{a+b-1}$. To preserve the normalization, we note that the number of such permutations is equal to the number of different label pairs; i. e., $N(N-1) / 2$. Thus the normalized, fully antisymmetric double-replacement symbolic state can be written as

$$
\left|\Psi_{d}\right\rangle=\sqrt{\frac{2}{N(N-1)}} \sum_{a<b}(-1)^{a+b-1}\left|\left\{\Psi_{\text {sub }}(1,2 \cdots \bar{a} \cdots \bar{b} \cdots N) J_{\text {sub }}, \psi_{v}(a, b) J_{v}\right\} J_{\text {tot }}\right\rangle .
$$

Here, $\bar{a}, \bar{b}$ show that the label ordering does not include $a, b$. Similarly we can write

$$
\left|\Psi_{s}\right\rangle=\sqrt{\frac{1}{N}} \sum_{a}(-1)^{N-a}\left|\left\{\Psi_{\text {sub }}(1,2 \cdots \bar{a} \cdots N) J_{\text {sub }}, \psi_{v}(a) j_{v}\right\} J_{\text {tot }}\right\rangle .
$$


By summing over all allowed permutations, taking advantage of (12) and (13), and using the fact that the correlation orbitals are orthogonal to all the reference and subconfiguration orbitals, we find that we need the following five different types of matrix elements of the operator G. In the next section we will consider each of these matrix elements in detail.

$$
\begin{aligned}
& \left\langle\Psi_{\mathrm{ref}}|\mathbf{G}| \Psi_{d}\right\rangle=\sqrt{\frac{N(N-1)}{2}}\left\langle\Psi_{\mathrm{ref}}\left|G_{N-1, N}\right| \Phi_{d}\right\rangle \\
& \left\langle\Psi_{\mathrm{ref}}|\mathbf{G}| \Psi_{s}\right\rangle=\frac{\sqrt{N}(N-1)}{2} \\
& \times\left[\left\langle\Psi_{\mathrm{ref}}\left|G_{N-1, N}\right| \Phi_{s}\right\rangle-\left\langle\Psi_{\mathrm{ref}}\left|G_{N-1, N}\right| \Phi_{s}^{X}\right\rangle\right] \\
& \left\langle\Psi_{d}^{\prime}|\mathbf{G}| \Psi_{d}\right\rangle=2(N-2) \times
\end{aligned}
$$

$$
\begin{aligned}
& \left\langle\Psi_{s}^{\prime}|\mathbf{G}| \Psi_{s}\right\rangle=(N-1) \\
& \times\left[\left\langle\Phi_{s}^{\prime}\left|G_{N-1, N}\right| \Phi_{s}\right\rangle-\left\langle\Phi_{s}^{\prime}\left|G_{N-1, N}\right| \Phi_{s}^{X}\right\rangle\right] \\
& +\frac{(N-1)(N-2)}{2}\left\langle\Phi_{s}^{\prime}\left|G_{N-2, N-1}\right| \Phi_{s}\right\rangle \\
& \left\langle\Psi_{s}^{\prime}|\mathbf{G}| \Psi_{d}\right\rangle=\sqrt{2(N-1)} \\
& \times\left[(N-2)\left\langle\Phi_{s}^{\prime}\left|G_{N-2, N-1}\right| \Phi_{d}\right\rangle+\left\langle\Phi_{s}^{\prime}\left|G_{N-1, N}\right| \Phi_{d}\right\rangle\right]
\end{aligned}
$$

\section{Matrix elements of form $\left\langle\Psi_{\text {ref }}|\mathbf{G}| \Psi_{d}\right\rangle$}

We start with the two-electron MCFP expansion of the reference state:

$$
\begin{aligned}
& \left.\left|\Psi_{\mathrm{ref}}(1,2 \cdots N) J_{\mathrm{tot}}\right\rangle=\sum_{\substack{\left(\Psi_{\text {sub }}^{\mathrm{ref}}\right) \\
\left(\psi_{2}^{\text {ref }}\right) J_{2}^{\text {ruf }}}}\left(\Psi_{\mathrm{ref}} \mid\right\} \Psi_{\text {sub }}^{\mathrm{ref}}, \psi_{2}^{\mathrm{ref}}\right) \\
& \times\left|\left\{\Psi_{\mathrm{sub}}^{\mathrm{ref}}(1 \cdots N-2) J_{\mathrm{sub}}^{\mathrm{ref}}, \psi_{2}^{\mathrm{ref}}(N-1, N) J_{2}^{\mathrm{ref}}\right\} J_{\text {tot }}\right\rangle .
\end{aligned}
$$

The sum here extends over all possible subconfigurations $\left(\Psi_{\text {sub }}^{\text {ref }}\right) J_{\text {sub }}^{\text {ref }}$ and pullout states $\left(\psi_{2}^{\text {ref }}\right) J_{2}^{\text {ref }}$. The subscript "2" here indicates that the pullout state is a two-electron state. Substituting (19) and (2) into (14), we obtain

$$
\begin{aligned}
& \left.\left\langle\Psi_{\text {ref }}|\mathbf{G}| \Psi_{d}\right\rangle=\sqrt{\frac{N(N-1)}{2}} \sum_{\substack{\left(\Psi_{\text {sub }}^{\text {ref }}()_{\text {sub }}^{\text {ref }},\left(\psi_{2}^{\text {ref }}\right) J_{2}^{\text {ref }}\right.}}\left(\Psi_{\text {ref }} \mid\right\}\left(\Psi_{\text {sub }}^{\text {ref }}\right) J_{\text {sub }}^{\text {ref }},\left(\psi_{2}^{\text {ref }}\right) J_{2}^{\text {ref }}\right) \\
& \times\left\langle\left\{\left(\Psi_{\text {sub }}^{\text {ref }}\right) J_{\text {sub }}^{\text {ref }},\left(\psi_{2}^{\text {ref }}\right) J_{2}^{\text {ref }}\right\} J_{\text {tot }}\left|G_{N-1, N}\right|\left\{\left(\Psi_{\text {sub }}\right) J_{\text {sub }},\left(\psi_{v}\right) J_{v}\right\} J_{\text {tot }}\right\rangle .
\end{aligned}
$$

It is important to keep in mind that the operator $G_{N-1, N}$ acts only on the two-electron states $\left(\psi_{2}^{\text {ref }}\right) J_{2}^{\text {ref }}$ and $\left(\psi_{v}\right) J_{v}$.

To isolate the two-electron matrix element, we uncouple the subconfiguration from the two-electron pair on both sides. Orthonormality selects a single term $\left(\Psi_{\text {sub }}\right) J_{\text {sub }}$ in the sum over $\left(\Psi_{\text {sub }}^{\text {ref }}\right) J_{\text {sub }}^{\text {ref }}$. We can simplify the sum over the pullout states by noting that the selected subconfiguration specifies the pullout orbitals uniquely. The total angular momentum of the pullout pair is fixed to $J_{v}$ by the scalar nature of the operator. Thus the sum over the pullout states $\left(\psi_{2}^{\text {ref }}\right) J_{2}^{\text {ref }}$ reduces to a single term as well. Applying the orthogonality relation for vector-coupling coefficients we get

$$
\left.\left\langle\Psi_{\text {ref }}|\mathbf{G}| \Psi_{d}\right\rangle=\sqrt{\frac{N(N-1)}{2}}\left(\Psi_{\text {ref }} \mid\right\}\left(\Psi_{\text {sub }}\right) J_{\text {sub }},\left(\psi_{2}^{\text {ref }}\right) J_{v}\right)\left\langle\left(\psi_{2}^{\text {ref }}\right) J_{v}|G|\left(\psi_{v}\right) J_{v}\right\rangle
$$


Note that the MCFP appearing here is closely related to an N-electron overlap

$$
\begin{aligned}
\left\langle\overline{\Psi_{d}} \mid \Psi_{\text {ref }}\right\rangle & \left.=\sqrt{\frac{N(N-1)}{2}}\left(\Psi_{\text {ref }} \mid\right\}\left(\Psi_{\text {sub }}\right) J_{\text {sub }},\left(\psi_{2}^{\text {ref }}\right) J_{v}\right) \\
\left|\overline{\Psi_{d}}\right\rangle & =\mathcal{A}_{N}\left|\left\{\left(\Psi_{\text {sub }}\right) J_{\text {sub }},\left(\psi_{2}^{\text {ref }}\right) J_{v}\right\} J_{\text {tot }}\right\rangle .
\end{aligned}
$$

Here $\mathcal{A}_{N}$ is the $N$-electron antisymmetrization operator; $\left|\overline{\Psi_{d}}\right\rangle$ is the same as $\left|\Psi_{d}\right\rangle$, but with $\left(\psi_{v}\right) J_{v}$ replaced by $\left(\psi_{2}^{\text {ref }}\right) J_{v}$, a two-electron state formed from the orbitals present in $\left|\Psi_{\text {ref }}\right\rangle$ and absent in $\left|\Psi_{d}\right\rangle$. Equation (23) is easily derived using (12) and the definition of the MCFP.

\section{Matrix elements of form $\left\langle\Psi_{\text {ref }}|\mathbf{G}| \Psi_{s}\right\rangle$}

We begin with Equation (16), and isolate the parts of the matrix element on which the operator $G_{N-1, N}$ acts, by doing two MCFP expansions: a two-electron expansion (19) of the reference state, and a one-electron expansion for the subconfiguration of the symbolic state

$$
\begin{aligned}
& \left|\Psi_{\text {sub }}(1 \cdots N-1) J_{\text {sub }}\right\rangle= \\
& \left.\quad \sum_{\substack{\left(\Psi^{\text {sub }}\right) J_{1}^{\text {sub }} \\
\left(\psi_{1}^{\text {sub }}\right) j_{s}}}\left(\Psi_{1}^{\text {sub }}, \psi_{1}^{\text {sub }} \mid\right\} \Psi_{\text {sub }}\right)\left|\left\{\Psi_{1}^{\text {sub }}(1 \cdots N-2) J_{1}^{\text {sub }}, \psi_{1}^{\text {sub }}(N-1) j_{s}\right\} J_{\text {sub }}\right\rangle .
\end{aligned}
$$

We recouple $\left|\Phi_{s}\right\rangle,\left|\Phi_{s}^{X}\right\rangle$ to form a state of two electrons with labels $N-1, N$, by use of

$$
\begin{aligned}
& \left|\left\{\left(J_{1}^{\text {sub }} j_{s}\right) J_{\text {sub }}, j_{v}\right\} J_{\text {tot }}\right\rangle= \\
& \sum_{J_{G}}\left\langle\left\{J_{1}^{\text {sub }},\left(j_{s} j_{v}\right) J_{G}\right\} J_{\text {tot }} \mid\left\{\left(J_{1}^{\text {sub }} j_{s}\right) J_{\text {sub }}, j_{v}\right\} J_{\text {tot }}\right\rangle\left|\left\{J_{1}^{\text {sub }},\left(j_{s} j_{v}\right) J_{G}\right\} J_{\text {tot }}\right\rangle= \\
& \sum_{J_{G}}(-1)^{j_{s}+j_{v}+J_{1}^{\text {sub }}+J_{\text {tot }}}\left[J_{\text {sub }}, J_{G}\right]^{1 / 2}\left\{\begin{array}{ccc}
J_{1}^{\text {sub }} & j_{s} & J_{\text {sub }} \\
j_{v} & J_{\text {tot }} & J_{G}
\end{array}\right\}\left|\left\{J_{1}^{\text {sub }},\left(j_{s} j_{v}\right) J_{G}\right\} J_{\text {tot }}\right\rangle .
\end{aligned}
$$

Here the recoupling coefficient has been written in terms of a $6 j$-symbol and we use the common notation $[a, b, \cdots]$ to stand for $(2 a+1)(2 b+1) \cdots$. Next we combine the contributions from $\left|\Phi_{s}\right\rangle$ and $\left|\Phi_{s}^{X}\right\rangle$ into an antisymmetric two-electron state

$$
\left|\left(\psi_{s v}\right) J_{G}\right\rangle=\sqrt{\frac{1}{2}}\left[\left|\left\{\psi_{1}^{\mathrm{sub}}(N-1) j_{s}, \psi_{v}(N) j_{v}\right\} J_{G}\right\rangle-\left|\left\{\psi_{1}^{\mathrm{sub}}(N) j_{s}, \psi_{v}(N-1) j_{v}\right\} J_{G}\right\rangle\right] .
$$

Now we can follow reasoning similar to that which led to (21). The result is

$$
\begin{aligned}
& \left\langle\Psi_{\text {ref }}|\mathbf{G}| \Psi_{s}\right\rangle=\frac{\sqrt{N}(N-1)}{\sqrt{2}} \sum_{\substack{\left(\Psi_{1}^{\text {sub }}\right) J_{1}^{\text {sub }} \\
j_{s}, J_{G}}}(-1)^{j_{s}+j_{v}+J_{1}^{\text {sub }}+J_{\text {tot }}}\left[J_{\text {sub }}, J_{G}\right]^{1 / 2}\left\{\begin{array}{ccc}
J_{1}^{\text {sub }} & j_{s} & J_{\text {sub }} \\
j_{v} & J_{\text {tot }} & J_{G}
\end{array}\right\} \\
& \times\left\langle\left(\psi_{2}^{\text {ref }}\right) J_{G}|\mathbf{G}|\left(\psi_{s v}\right) J_{G}\right\rangle\left(\Psi_{\text {ref }}\left\{\mid\left(\Psi_{1}^{\text {sub }}\right) J_{1}^{\text {sub }},\left(\psi_{2}^{\text {ref }}\right) J_{G}\right)\left(\left(\Psi_{1}^{\text {sub }}\right) J_{1}^{\text {sub }},\left(\psi_{1}^{\text {sub }}\right) j_{s} \mid\right\} \Psi_{\text {sub }}\right) .
\end{aligned}
$$

Here, $j_{s}$ runs over all the orbitals of the $(N-1)$-electron symbolic-state subconfiguration $\Psi_{\text {sub. }}$ Orthogonality of the subconfigurations eliminates the sum over $\Psi_{\text {sub }}^{\text {ref }}$. The two-electron state $\psi_{2}^{\text {ref }}$ contains orbitals present in $\Psi_{\text {ref }}$, but absent from $\Psi_{1}^{\text {sub }}$.

Alternatively we can rewrite this result by replacing the MCFP coefficients with overlaps between antisymmetric states. Using

$$
\left.\left\langle\left\{\left(\Psi_{1}^{\text {sub }}\right) J_{1}^{\text {sub }},\left(\psi_{1}^{\text {sub }}\right) j_{s}\right\} J_{\text {sub }} \mid\left(\Psi_{\text {sub }}\right) J_{\text {sub }}\right\rangle=\sqrt{N-1}\left(\left(\Psi_{1}^{\text {sub }}\right) J_{1}^{\text {sub }},\left(\psi_{1}^{\text {sub }}\right) j_{s} \mid\right\} \Psi_{\text {sub }}\right)
$$


and a relation similar to (22), we obtain

$$
\begin{aligned}
& \left\langle\Psi_{\text {ref }}|\mathbf{G}| \Psi_{s}\right\rangle=\sum_{\substack{\left(\Psi_{1}^{\text {sub }}\right) J_{1}^{\text {sub }} \\
j_{s}, J_{G}}}(-1)^{j_{s}+j_{v}+J_{1}^{\text {sub }}+J_{\text {tot }}}\left[J_{\text {sub }}, J_{G}\right]^{1 / 2}\left\{\begin{array}{ccc}
J_{1}^{\text {sub }} & j_{s} & J_{\text {sub }} \\
j_{v} & J_{\text {tot }} & J_{G}
\end{array}\right\}\left\langle\left(\psi_{2}^{\text {ref }}\right) J_{G}|\mathbf{G}|\left(\psi_{s v}\right) J_{G}\right\rangle \\
& \times\left\langle\left(\Psi_{\text {ref }}\right) J_{\text {tot }} \mid\left\{\left(\Psi_{1}^{\text {sub }}\right) J_{1}^{\text {sub }},\left(\psi_{2}^{\text {ref }}\right) J_{G}\right\} J_{\text {tot }}\right\rangle\left\langle\left\{\left(\Psi_{1}^{\text {sub }}\right) J_{1}^{\text {sub }},\left(\psi_{1}^{\text {sub }}\right) j_{s}\right\} J_{\text {sub }} \mid\left(\Psi_{\text {sub }}\right) J_{\text {sub }}\right\rangle .
\end{aligned}
$$

\section{Matrix elements of form $\left\langle\Psi_{d}^{\prime}|\mathbf{G}| \Psi_{d}\right\rangle$}

For matrix elements connecting double-replacement symbolic states we start with (16):

$$
\begin{gathered}
\quad\left\langle\Psi_{d}^{\prime}|\mathbf{G}| \Psi_{d}\right\rangle=\mathcal{M}_{v v}+\mathcal{M}_{s s}+\mathcal{M}_{s v}, \\
\mathcal{M}_{v v}=\left\langle\Phi_{d}^{\prime}\left|G_{N-1, N}\right| \Phi_{d}\right\rangle, \\
\mathcal{M}_{s s}=\frac{(N-3)(N-2)}{2}\left\langle\Phi_{d}^{\prime}\left|G_{N-3, N-2}\right| \Phi_{d}\right\rangle \\
\mathcal{M}_{s v}=2(N-2)\left[\left\langle\Phi_{d}^{\prime}\left|G_{N-2, N-1}\right| \Phi_{d}\right\rangle-\left\langle\Phi_{d}^{\prime}\left|G_{N-2, N-1}\right| \Phi_{d}^{X}\right\rangle\right] .
\end{gathered}
$$

Here, $\mathcal{M}_{s s}, \mathcal{M}_{v v}$ represent interactions within subconfigurations and correlation pairs, respectively, and $\mathcal{M}_{s v}$ describes the interaction between a subconfiguration and a correlation pair. The first two terms, $\mathcal{M}_{s s}$ and $\mathcal{M}_{v v}$, are almost trivial. We uncouple the subconfigurations from the pairs, and then take into account the orthogonality of the functions on which the operator $\mathbf{G}$ does not act and of the vector-coupling coefficients that appear upon uncoupling the subconfigurations from the pairs. The results are

$$
\begin{aligned}
& \mathcal{M}_{v v}=\left\langle\left(\Psi_{\text {sub }}^{\prime}\right) J_{\text {sub }}^{\prime} \mid\left(\Psi_{\text {sub }}\right) J_{\text {sub }}\right\rangle\left\langle\left(\psi_{v}^{\prime}\right) J_{v}^{\prime}|\mathbf{G}|\left(\psi_{v}\right) J_{v}\right\rangle, \\
& \mathcal{M}_{s s}=\left\langle\left(\Psi_{\text {sub }}^{\prime}\right) J_{\text {sub }}^{\prime}|\mathbf{G}|\left(\Psi_{\text {sub }}\right) J_{\text {sub }}\right\rangle\left\langle\left(\psi_{v}^{\prime}\right) J_{v}^{\prime} \mid\left(\psi_{v}\right) J_{v}\right\rangle .
\end{aligned}
$$

Dealing with $\mathcal{M}_{s v}$ requires more effort. We use the tensor expansion (5) for the scalar operator $\mathbf{G}=\sum G_{i j}$. We represent the nature of angular momentum coupling in the $\mathcal{M}_{s v}$ term by the use of JB graphs [2]. We focus on the part of the $N$-electron matrix element that depends upon the correlation orbitals and the operator:

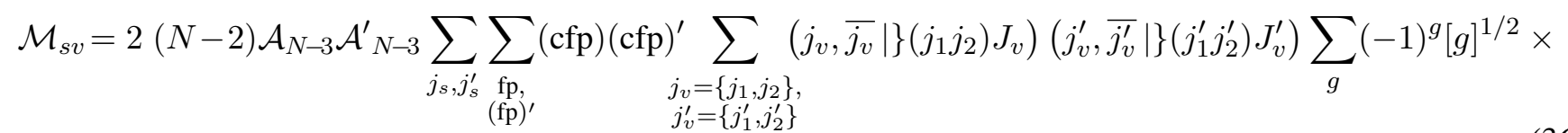
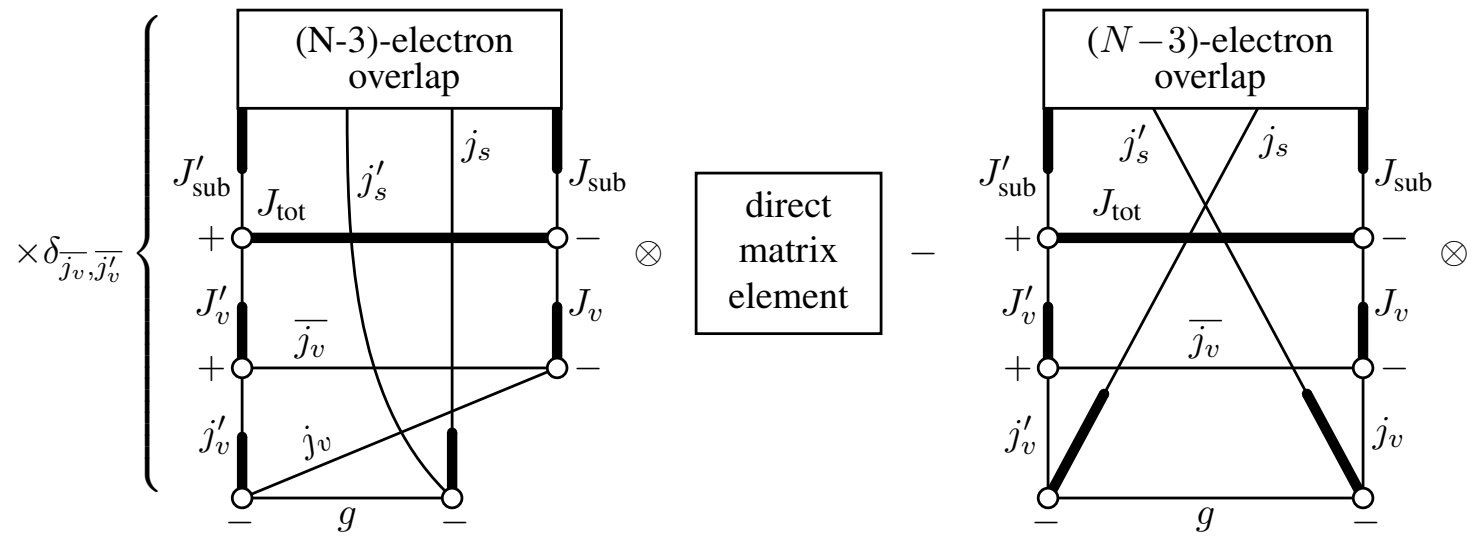
Here, $j_{s}, j_{s}^{\prime}$ are subconfiguration orbitals; $j_{s}$ and $j_{s}^{\prime}$ run over all the subconfiguration subshells. Coefficients of fractional parentage occurring upon separating one $j_{s}$ or $j_{s}^{\prime}$ electron from its subshell are denoted as (cfp), (cfp) $)^{\prime}$, and the fractional parents are labeled $\mathrm{fp}, \mathrm{fp}^{\prime}$. Operators $\mathcal{A}_{N-3}, \mathcal{A}_{N-3}^{\prime}$ complete the antisymmetrization of the subconfigurations by taking into account allowed permutations of electron labels, with the last label kept in the $j_{s}, j_{s}^{\prime}$ subshell. The sums over the correlation orbitals are indexed by $j_{v}, j_{v}^{\prime}$, and there are at most two values for each of these orbitals, namely, $j_{1}, j_{2}$ or $j_{1}^{\prime}, j_{2}^{\prime}$. Noninteracting electrons in correlation orbitals are labeled $\overline{j_{v}}, \overline{j_{v}^{\prime}}$; that is, if $j_{v}=j_{1}$, then $\overline{j_{v}}=j_{2}$, and so forth. The MCFP for the correlation pair is represented by $\left.\left(j_{v}, \overline{j_{v}} \mid\right\}\left(j_{1} j_{2}\right) J_{v}\right)$. This coefficient preserves the coupling order in the correlation pair: for both permutations of electron labels $(N-1, N)$, the noninteracting electron is coupled second.

Direct and exchange matrix element boxes contain radial integrals and reduced matrix elements of oneelectron tensor operators:

$$
\begin{aligned}
& \begin{array}{c}
\begin{array}{c}
\text { direct } \\
\text { matrix } \\
\text { element }
\end{array} \\
=
\end{array}=\frac{1}{\left[j_{v}^{\prime}, j_{s}, g\right]^{1 / 2}} \\
& \times\left\langle j_{v}^{\prime}\left|G^{(g)}\right| j_{v}\right\rangle\left\langle j_{s}^{\prime}\left|G^{(g)}\right| j_{s}\right\rangle R_{g}^{\text {direct }},
\end{aligned}
$$

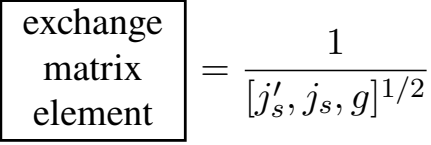

$$
\begin{aligned}
& \times\left\langle j_{v}^{\prime}\left|G^{(g)}\right| j_{s}\right\rangle\left\langle j_{s}^{\prime}\left|G^{(g)}\right| j_{v}\right\rangle R_{g}^{\text {exchange }} .
\end{aligned}
$$

Here, $R_{g}^{\text {direct }}, R_{g}^{\text {exchange }}$ are scalar two-electron functions (radial integrals), and the standard reduced matrix elements have been defined as in Cowan [14]. To derive explicit dependence of $\mathcal{M}_{s v}$ on the quantum numbers of correlation orbitals, we proceed in three steps: first, we extract the two-electron matrix element; next, we isolate the subconfiguration contribution; and last, we compute the remaining purely angular factors.

Two-electron matrix element. Each JB graph in (37) can be cut on four lines $j_{v}^{\prime}, j_{v}, j_{s}^{\prime}, j_{s}$ to separate the operator-dependent part. We notice that the matrix element of $\mathbf{G}$ between coupled, antisymmetric, and nonequivalent two-electron states has the diagrammatic form

$$
\left\langle\left(j_{s}^{\prime}, j_{v}^{\prime}\right) J_{G}|\mathbf{G}|\left(j_{s}, j_{v}\right) J_{G}\right\rangle=\sum_{g}(-1)^{g}[g]^{1 / 2}
$$




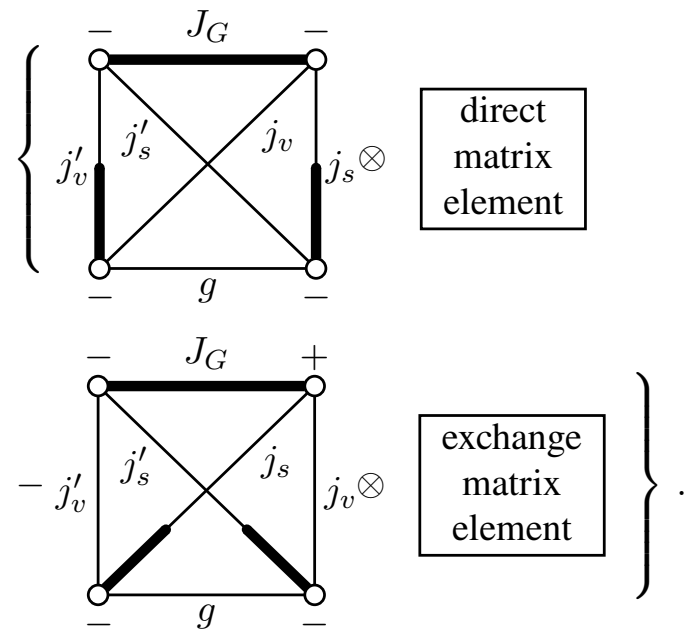

Thus we can factor out the tensor expansion and so reduce (37) to

$$
\mathcal{M}_{s v}=2(N-2) \mathcal{A}_{N-3} \mathcal{A}^{\prime}{ }_{N-3} \sum_{j_{s}, j_{s}^{\prime}} \sum_{\substack{\mathrm{fp} \\(\mathrm{fp})^{\prime}}}(\mathrm{cfp})(\mathrm{cfp})^{\prime}
$$

$\left.\left.\times \sum_{j_{v}, j_{v}^{\prime}}\left(j_{v}, \overline{j_{v}} \mid\right\}\left(j_{1} j_{2}\right) J_{v}\right)\left(j_{v}^{\prime}, \overline{j_{v}^{\prime}} \mid\right\}\left(j_{1}^{\prime} j_{2}^{\prime}\right) J_{v}^{\prime}\right) \sum_{J_{G}} \delta_{\overline{j_{v}}, \overline{j_{v}^{\prime}}}$ $\times(-1)^{2 j_{v}} \frac{\left[J_{G}, J_{v}\right]^{1 / 2}}{\left[j_{s}, \overline{j_{v}}\right]^{1 / 2}}\left\langle\left(j_{s}^{\prime}, j_{v}^{\prime}\right) J_{G}|\mathbf{G}|\left(j_{s}, j_{v}\right) J_{G}\right\rangle \times$

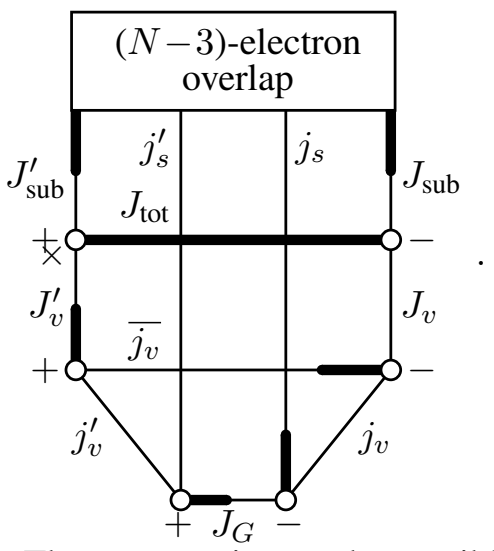

The new sum is over the possible two-electron quantum numbers $J_{G}$ that result as the orbitals $j_{s}, j_{v}$ and $j_{s}^{\prime}, j_{v}^{\prime}$ are coupled in pairs.

Contribution of subconfigurations. To isolate the subconfigurations, we cut the diagram of (40) on four lines $J_{\text {sub }}^{\prime}, j_{s}^{\prime}, j_{s}, J_{\text {sub. This yields }}$

$$
\begin{aligned}
& \mathcal{M}_{s v}=2 \sum_{j_{s}, j_{s}^{\prime}} \sum_{k} \Gamma_{k}\left(\Psi_{\mathrm{sub}}^{\prime}, j_{s}^{\prime} ; \Psi_{\mathrm{sub}}, j_{s}\right) \\
& \left.\left.\times \sum_{j_{v}, j_{v}^{\prime}}\left(j_{v}, \overline{j_{v}} \mid\right\}\left(j_{1} j_{2}\right) J_{v}\right)\left(j_{v}^{\prime}, \overline{j_{v}^{\prime}} \mid\right\}\left(j_{1}^{\prime} j_{2}^{\prime}\right) J_{v}^{\prime}\right)
\end{aligned}
$$

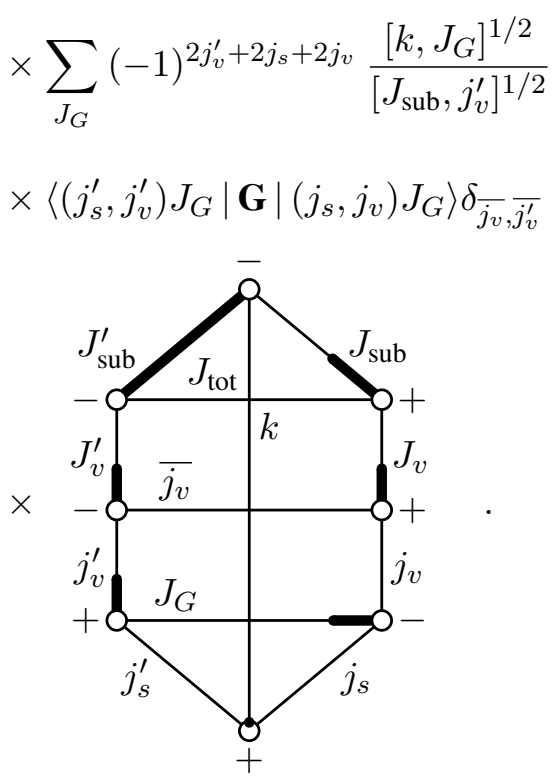

The values of $k$ in this sum are limited by a triangle relation $\left(j_{s}^{\prime} k j_{s}\right)$. The quantity $\Gamma_{k}\left(\Psi_{\text {sub }}^{\prime}, j_{s}^{\prime} ; \Psi_{\text {sub }}, j_{s}\right)$ in (41) depends on all the internal couplings required to specify the two subconfigurations, and does not depend on the quantum numbers of correlation orbitals:

$$
\Gamma_{k}\left(\Psi_{\text {sub }}^{\prime}, j_{s}^{\prime} ; \Psi_{\text {sub }}, j_{s}\right)=(N-2) \frac{\left[J_{\text {sub }}^{\prime}\right]^{1 / 2}}{\left[j_{s}^{\prime}\right]^{1 / 2}} \mathcal{A}_{N-3}
$$

$$
\times \mathcal{A}^{\prime}{ }_{N-3} \sum_{\substack{\mathrm{fp},(\mathrm{fp})^{\prime}}}(\mathrm{cfp})(\mathrm{cfp})^{\prime}
$$

The quantity $\Gamma_{k}$ can also be seen in another context. Consider the matrix element of a one-body operator of rank $k$,

$$
W_{q}^{(k)}=\sum_{i=1}^{N_{\text {sub }}} w_{q}^{(k)}(i)
$$

between two subconfigurations $\left|\left(\Psi_{\text {sub }}\right) J_{\text {sub }}\right\rangle$ and $\left|\left(\Psi_{\text {sub }}^{\prime}\right) J_{\text {sub }}^{\prime}\right\rangle$ of $N_{\text {sub }}$ electrons. The graphical analysis now yields a formula which can be used to compute $\Gamma_{k}$ as needed,

$$
\begin{aligned}
& \left\langle\left(\Psi_{\mathrm{sub}}^{\prime}\right) J_{\mathrm{sub}}^{\prime}\left|W^{(k)}\right|\left(\Psi_{\mathrm{sub}}\right) J_{\mathrm{sub}}\right\rangle= \\
& \sum_{j_{s}^{\prime}, j_{s}} \Gamma_{k}\left(\Psi_{\mathrm{sub}}^{\prime}, j_{s}^{\prime} ; \Psi_{\mathrm{sub}}, j_{s}\right)\left\langle j_{s}^{\prime}\left|w^{(k)}\right| j_{s}\right\rangle .
\end{aligned}
$$


Remaining angular factors. We still have to work out an algebraic expression for the diagram of (41). By straightforward application of the reduction rules for JB graphs, we get

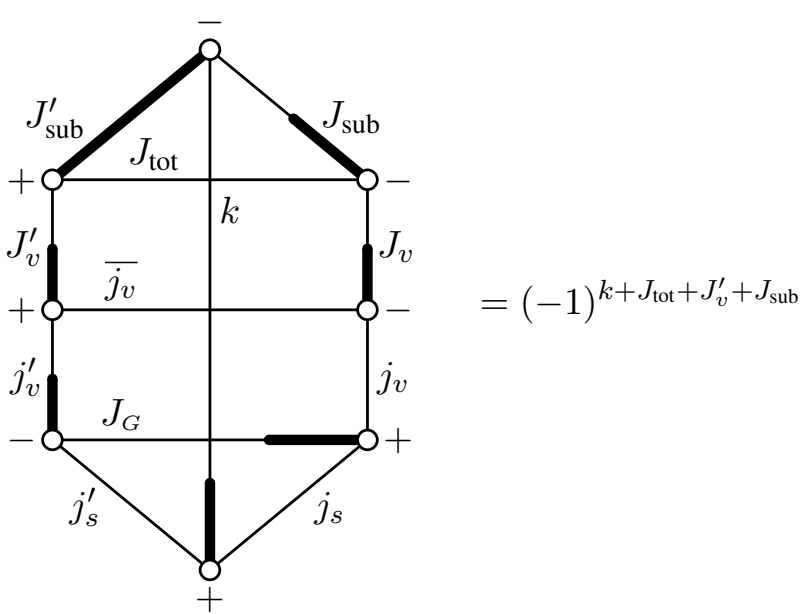

$$
\begin{aligned}
& \times(-1)^{\overline{j_{v}}+J_{v}+j_{s}-J_{G}}\left[k, J_{G}, j_{v}^{\prime}, J_{v}, J_{v}^{\prime}, J_{\mathrm{sub}}\right]^{1 / 2} \\
& \times\left\{\begin{array}{ccc}
J_{v} & k & J_{v}^{\prime} \\
J_{\text {sub }}^{\prime} & J_{\text {tot }} & J_{\text {sub }}
\end{array}\right\}\left\{\begin{array}{lll}
J_{v} & k & J_{v}^{\prime} \\
j_{v}^{\prime} & j_{v} & j_{v}
\end{array}\right\}\left\{\begin{array}{lll}
j_{s} & k & j_{s}^{\prime} \\
j_{v}^{\prime} & J_{G} & j_{v}
\end{array}\right\}
\end{aligned}
$$

Final results. Now we are ready to write the final expression:

$$
\mathcal{M}_{s v}=\sum_{j_{s}, j_{s}^{\prime}} \sum_{j_{v}, j_{v}^{\prime}} \sum_{J_{G}} \mathcal{C}_{\mathrm{sym}}\left\langle\left(j_{s}^{\prime}, j_{v}^{\prime}\right) J_{G}|\mathbf{G}|\left(j_{s}, j_{v}\right) J_{G}\right\rangle,
$$

where

$$
\begin{aligned}
& \left.\left.\mathcal{C}_{\text {sym }}=2 \delta_{\overline{j_{v}}, \overline{j_{v}^{\prime}}}\left(j_{v}, \overline{j_{v}} \mid\right\}\left(j_{1} j_{2}\right) J_{v}\right)\left(j_{v}^{\prime}, \overline{j_{v}^{\prime}} \mid\right\}\left(j_{1}^{\prime} j_{2}^{\prime}\right) J_{v}^{\prime}\right) \\
& \times \sum_{k} \Gamma_{k}\left(\Psi_{\text {sub }}^{\prime}, j_{s}^{\prime} ; \Psi_{\text {sub }}, j_{s}\right)\left[J_{G}, k\right]\left[J_{v}, J_{v}^{\prime}\right]^{1 / 2} \\
& \times(-1)^{k+J_{\text {tot }}+J_{v}^{\prime}+J_{\text {sub }}+\overline{j_{v}}+J_{v}-j_{s}-J_{G}} \\
& \times\left\{\begin{array}{ccc}
J_{v} & k & J_{v}^{\prime} \\
J_{\text {sub }}^{\prime} & J_{\text {tot }} & J_{\text {sub }}
\end{array}\right\}\left\{\begin{array}{lll}
J_{v} & k & J_{v}^{\prime} \\
j_{v}^{\prime} & \overline{j_{v}} & j_{v}
\end{array}\right\}\left\{\begin{array}{lll}
j_{s} & k & j_{s}^{\prime} \\
j_{v}^{\prime} & J_{G} & j_{v}
\end{array}\right\} .
\end{aligned}
$$

The expression is rather complicated in appearance, but it is completely general and convenient to use in a computer program.

In summary, by expanding an $N$-electron matrix element in terms of two-electron matrix elements, we obtained explicit dependence of the matrix element $\left\langle\Psi_{d}^{\prime}|\mathbf{G}| \Psi_{d}\right\rangle$ on the quantum numbers of the correlation orbitals.

\section{Matrix elements connecting single-replacement symbolic states}

Next we consider the matrix elements connecting single-replacement symbolic states. To derive the dependence of the matrix element (17) on the correlation orbitals, we retrace our steps taken in the treatment of the interaction between double-replacement symbolic states. Without repeating the details, we state the final result

$$
\left\langle\Psi_{s}^{\prime}|\mathbf{G}| \Psi_{s}\right\rangle=\mathcal{M}_{s s}+\mathcal{M}_{s v}
$$

where

$$
\begin{aligned}
\mathcal{M}_{s s} & =\left\langle\left(\Psi_{\text {sub }}^{\prime}\right) J_{\text {sub }}^{\prime}|\mathbf{G}|\left(\Psi_{\text {sub }}\right) J_{\text {sub }}\right\rangle \\
& \times\left\langle\left(\psi_{v}^{\prime}\right) j_{v}^{\prime} \mid\left(\psi_{v}\right) j_{v}\right\rangle \\
\mathcal{M}_{s v} & =\sum_{\substack{j_{s}, j_{s}^{\prime} \\
k, J_{G}}}(-1)^{J_{\text {tot }}+J_{\text {sub }}-J_{G}-j_{s}}\left[J_{G}, k\right] \\
& \times\left\{\begin{array}{ccc}
J_{\text {sub }} & k & J_{\text {sub }}^{\prime} \\
j_{v}^{\prime} & J_{\text {tot }} & j_{v}
\end{array}\right\}\left\{\begin{array}{lll}
j_{v} & k & j_{v}^{\prime} \\
j_{s}^{\prime} & J_{G} & j_{s}
\end{array}\right\} \Gamma_{k}\left(\Psi_{\text {sub }}^{\prime}, j_{s}^{\prime} ; \Psi_{\text {sub }}, j_{s}\right) \\
& \times\left\langle\left(j_{s}^{\prime}, j_{v}^{\prime}\right) J_{G}|\mathbf{G}|\left(j_{s}, j_{v}\right) J_{G}\right\rangle .
\end{aligned}
$$

Again, the possible values of $J_{G}$ are limited by triangle relations $\left(j_{s} j_{v} J_{G}\right)$ and $\left(j_{s}^{\prime} j_{v}^{\prime} J_{G}\right)$, the values of $\mathrm{k}$ are defined by $\left(j_{s} k j_{s}^{\prime}\right)$, and $j_{s}, j_{s}^{\prime}$ run over all orbitals of their corresponding subconfigurations. The definition of $\Gamma_{k}\left(\Psi_{\text {sub }}^{\prime}, j_{s}^{\prime} ; \Psi_{\text {sub }}, j_{s}\right)$ is given in (44).

\section{Matrix elements connecting the two types of symbolic states}

To complete our discussion on the dependence of the matrix elements (14) - (18) upon correlation orbitals, we examine the matrix element $\left\langle\Psi_{s}^{\prime}|\mathbf{G}| \Psi_{d}\right\rangle$ between a single-replacement symbolic state and a double-replacement symbolic state. We start with (18)

$$
\left\langle\Psi_{s}^{\prime}|\mathbf{G}| \Psi_{d}\right\rangle=\mathcal{M}_{s v v v}+\mathcal{M}_{v s s s},
$$

where

$\mathcal{M}_{s v v v}=\sqrt{2(N-1)}\left\langle\Phi_{s}^{\prime}\left|G_{N-1, N}\right| \Phi_{d}\right\rangle$,

$\mathcal{M}_{\text {vsss }}=(N-2) \sqrt{2(N-1)}\left\langle\Phi_{s}^{\prime}\left|G_{N-2, N-1}\right| \Phi_{d}\right\rangle$.

The $\mathcal{M}_{\text {svvv }}$ term connects three correlation orbitals and one subconfiguration orbital, whereas $\mathcal{M}_{\text {vsss }}$ connects three subconfiguration orbitals and one correlation orbital. 
The $\mathcal{M}_{\text {svvv }}$ term can be handled in a manner similar to that which we used in Section 6 to study the matrix element between a single-replacement symbolic state and a reference state. We transform $\left|\Phi_{s}^{\prime}\right\rangle$ using (24) and (25), and isolate an electron pair on which the operator acts. We also observe that

$$
\begin{aligned}
& \left\langle\left(\psi_{s v}^{\prime}\right) J_{v}|\mathbf{G}|\left(\psi_{v}\right) J_{v}\right\rangle= \\
& \sqrt{2}\left\langle\left\{\psi_{1}^{\prime \text { sub }}(N-1) j_{s}^{\prime}, \psi_{v}^{\prime}(N) j_{v}^{\prime}\right\} J_{v}|\mathbf{G}|\left(\psi_{v}\right) J_{v}\right\rangle,
\end{aligned}
$$

where $\left(\psi_{s v}^{\prime}\right)$ is an antisymmetric two-electron state defined as in (26). With this in mind, we obtain

$$
\begin{aligned}
& \mathcal{M}_{\text {svvv }}= \\
& \sqrt{N-1}\left(\left(\Psi_{\text {sub }}^{\prime}\right) J_{\text {sub }}^{\prime}\left\{\mid\left(\Psi_{\text {sub }}\right) J_{\text {sub }},\left(\psi_{1}^{\prime \text { sub }}\right) j_{s}^{\prime}\right)\right.
\end{aligned}
$$

$$
\begin{aligned}
& \times\left\langle\left\{J_{\text {sub }},\left(j_{s}^{\prime} j_{v}^{\prime}\right) J_{v}\right\} J_{\text {tot }} \mid\left\{\left(J_{\text {sub }} j_{s}^{\prime}\right) J_{\text {sub }}^{\prime}, j_{v}^{\prime}\right\} J_{\text {tot }}\right\rangle \\
& \times\left\langle\left(\psi_{s v}^{\prime}\right) J_{v}|\mathbf{G}|\left(\psi_{v}\right) J_{v}\right\rangle .
\end{aligned}
$$

Here, all primed quantities pertain to the single-replacement symbolic state, and unprimed quantities belong to the double-replacement symbolic state. The orbital $\left(\psi_{1}^{\prime \text { sub }}\right) j_{s}^{\prime}$ is present in $\Psi_{\text {sub }}^{\prime}$, but absent from $\Psi_{\text {sub }}$.

Next we turn our attention to the $\mathcal{M}_{\text {vsss }}$ term. Just as we did before, we isolate the electron pair on which the operator acts; in this case, the pair with electron labels $N-2, N-1$. To accomplish this, we do three MCFP expansions: one for each subconfiguration, and one for the correlation pair of the double-replacement symbolic state. Although similar expansions have been

used in previous sections, we repeat the equations for clarity of notation. In particular, the subconfiguration of the single-replacement symbolic state is expressed as

$$
\begin{aligned}
\left|\Psi_{\text {sub }}^{\prime}(1 \cdots N-1) J_{\text {sub }}^{\prime}\right\rangle= & \left.\sum_{\substack{\left(\Psi_{N-3}^{\prime}\right) J_{N-3}^{\prime},\left(\psi_{2}^{\prime \text { sub }}\right) J_{s}^{\prime}}}\left(\left(\Psi_{N-3}^{\prime}\right) J_{N-3}^{\prime},\left(\psi_{2}^{\prime \text { sub }}\right) J_{s}^{\prime} \mid\right\}\left(\Psi_{\text {sub }}^{\prime}\right) J_{\text {sub }}^{\prime}\right) \\
& \times\left|\left\{\Psi_{N-3}^{\prime}(1 \cdots N-3) J_{N-3}^{\prime}, \psi_{2}^{\prime \text { sub }}(N-2, N-1) J_{s}^{\prime}\right\} J_{\text {sub }}^{\prime}\right\rangle,
\end{aligned}
$$

where $\left(\psi_{2}^{\prime \text { sub }}\right) J_{s}^{\prime}$ is a coupled and antisymmetric pair of subconfiguration electrons.

The subconfiguration of the double-replacement symbolic state becomes

$$
\begin{aligned}
\left|\Psi_{\text {sub }}(1 \cdots N-2) J_{\text {sub }}\right\rangle= & \left.\sum_{\begin{array}{c}
\left(\Psi_{N-3}\right) J_{N-3} \\
\left(\psi_{1}^{\text {sub }}\right) j_{s}
\end{array}}\left(\left(\Psi_{N-3}\right) J_{N-3},\left(\psi_{1}^{\text {sub }}\right) j_{s} \mid\right\}\left(\Psi_{\text {sub }}\right) J_{\text {sub }}\right) \\
& \times\left|\left\{\Psi_{N-3}(1 \cdots N-3) J_{N-3}, \psi_{1}^{\text {sub }}(N-2) j_{s}\right\} J_{\text {sub }}\right\rangle
\end{aligned}
$$

where $\left(\psi_{1}^{\text {sub }}\right) j_{s}$ is a selected orbital from the subconfiguration of the double-replacement symbolic state.

The third MCFP expansion, for the correlation pair, is

$$
\left.\left|\psi_{v}(N-1, N) J_{v}\right\rangle=\sum_{j_{v}=\left\{j_{1}, j_{2}\right\}}\left(j_{v}, \overline{j_{v}} \mid\right\}\left(j_{1} j_{2}\right) J_{v}\right)\left|\left\{\psi_{v}(N-1) j_{v}, \overline{\psi_{v}}(N) \overline{j_{v}}\right\} J_{v}\right\rangle .
$$

Here, $j_{v}$ runs over the two orbitals of $\left|\left(\psi_{v}\right) J_{v}\right\rangle$, and $\overline{j_{v}}$ is defined as the other correlation orbital: if $j_{v}=j_{1}$, then $\overline{j_{v}}=j_{2}$, and vice versa. The relation (58) ensures that the electron labeled $N-1$ is the first in the coupling order of the correlation pair.

Having separated electrons with labels $N-2, N-1$ from their subconfigurations, we still need to couple the active electrons together on the right-hand side of the matrix element. This is conveniently accomplished by use of the recoupling relation

$$
\begin{aligned}
& \left|\left\{\left(J_{N-3} j_{s}\right) J_{\text {sub }},\left(j_{v}, \overline{j_{v}}\right) J_{v}\right\} J_{\text {tot }}\right\rangle=\sum_{J_{G}, J_{\text {int }}}\left|\left\{\left[J_{N-3},\left(j_{s} j_{v}\right) J_{G}\right] J_{\text {int }}, \overline{j_{v}}\right\} J_{\text {tot }}\right\rangle \\
& \times\left\langle\left\{\left[J_{N-3},\left(j_{s} j_{v}\right) J_{G}\right] J_{\text {int }}, \overline{j_{v}}\right\} J_{\text {tot }} \mid\left\{\left(J_{N-3} j_{s}\right) J_{\text {sub }},\left(j_{v}, \overline{j_{v}}\right) J_{v}\right\} J_{\text {tot }}\right\rangle .
\end{aligned}
$$


Notice that this step yields the desired electron pair $\left(j_{s}(N-2) j_{v}(N-1)\right) J_{G}$, which is coupled, but not antisymmetric.

Substituting (56) - (59) into (53) and keeping in mind (55), we get

$$
\begin{aligned}
& \left.\mathcal{M}_{v s s s}=(N-2) \sqrt{N-1} \sum_{\substack{\left(\Psi_{N-3}\right) J_{N-3}, j_{v}=\left\{j_{1}, j_{2}\right\} \\
\left(\psi_{2}^{\prime \text { sub }}\right) J_{G}}}\left(j_{v}, \overline{j_{v}} \mid\right\}\left(j_{1} j_{2}\right) J_{v}\right)\left\langle\left(\psi_{v}^{\prime}\right) j_{v}^{\prime} \mid\left(\overline{\psi_{v}}\right) \overline{j_{v}}\right\rangle \\
& \times\left(\left(\Psi_{\text {sub }}^{\prime}\right) J_{\text {sub }}^{\prime}\left\{\mid\left(\Psi_{N-3}\right) J_{N-3},\left(\psi_{2}^{\prime \text { sub }}\right) J_{G}\right)\left(\left(\Psi_{N-3}\right) J_{N-3},\left(\psi_{1}^{\text {sub }}\right) j_{s} \mid\right\}\left(\Psi_{\text {sub }}\right) J_{\text {sub }}\right) \\
& \times\left\langle\left\{\left[J_{N-3},\left(j_{s} j_{v}\right) J_{G}\right] J_{\text {sub }}^{\prime}, \overline{j_{v}}\right\} J_{\text {tot }} \mid\left\{\left(J_{N-3} j_{s}\right) J_{\text {sub }},\left(j_{v}, \overline{j_{v}}\right) J_{v}\right\} J_{\text {tot }}\right\rangle\left\langle\left(\psi_{2}^{\prime \text { sub }}\right) J_{G}|\mathbf{G}|\left(\psi_{s v}\right) J_{G}\right\rangle .
\end{aligned}
$$

Here, $\left(\psi_{s v}\right) J_{G}$ is a coupled and antisymmetric two-electron state as in (26), the orthogonality of Dirac orbitals requires that the two $(N-3)$-electron states on both sides of the matrix element be the same, and the scalar nature of the operator sets $J_{s}^{\prime}=J_{G}$ and $J_{\text {int }}=J_{\text {sub }}^{\prime}$. Also notice that for the $\mathcal{M}_{s v}$ term to have a nonzero value, the two correlation orbitals on which the operator does not act have to be the same.

The recoupling coefficient is easily evaluated using JB graphs:

$$
\left\langle\left\{\left[J_{N-3},\left(j_{s} j_{v}\right) J_{G}\right] J_{\text {sub }}^{\prime}, \overline{j_{v}}\right\} J_{\text {tot }} \mid\left\{\left(J_{N-3} j_{s}\right) J_{\text {sub }},\left(j_{v} \overline{j_{v}}\right) J_{v}\right\} J_{\text {tot }}\right\rangle=
$$

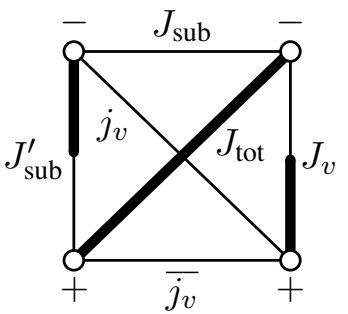

Expressing the MCFP coefficients in terms of overlaps between antisymmetric states, we can write the final results for the interaction between a single-replacement and a double-replacement symbolic state as

$$
\begin{aligned}
& \mathcal{M}_{\text {svvv }}=(-1)^{J_{\text {sub }}+j_{s}^{\prime}+j_{v}^{\prime}+J_{\text {tot }}}\left[J_{\text {sub }}^{\prime}, J_{v}\right]^{1 / 2} \\
& \times\left\{\begin{array}{ccc}
J_{\text {sub }} & j_{s}^{\prime} & J_{\text {sub }}^{\prime} \\
j_{v}^{\prime} & J_{\text {tot }} & J_{v}
\end{array}\right\} \quad\left\langle\left(\psi_{s v}^{\prime}\right) J_{v}|\mathbf{G}|\left(\psi_{v}\right) J_{v}\right\rangle \\
& \times\left\langle\left\{\left(\Psi_{\text {sub }}\right) J_{\text {sub }}, j_{s}^{\prime}\right\} J_{\text {sub }}^{\prime} \mid\left(\Psi_{\text {sub }}^{\prime}\right) J_{\text {sub }}^{\prime}\right\rangle,
\end{aligned}
$$

$$
\begin{aligned}
& \mathcal{M}_{v s s s}=\sqrt{2} \\
& \times \sum_{\substack{\left(\Psi_{N-3)}\right) J_{N-3} \\
\left(\psi_{2}^{\prime}\right) J_{G}}}\left\langle\left\{\left(\Psi_{N-3}\right) J_{N-3},\left(\psi_{2}^{\prime}\right) J_{G}\right\} J_{\text {sub }}^{\prime} \mid\left(\Psi_{\text {sub }}^{\prime}\right) J_{\text {sub }}^{\prime}\right\rangle \\
& \times\left\langle\left\{\left(\Psi_{N-3}\right) J_{N-3},\left(\psi_{1}^{\text {sub }}\right) j_{s}\right\} J_{\text {sub }} \mid\left(\Psi_{\text {sub }}\right) J_{\text {sub }}\right\rangle \\
& \left.\left.\times \sum_{j_{v}=\left\{j_{1}, j_{2}\right\}}\left\langle\left(\psi_{v}^{\prime}\right) j_{v}^{\prime} \mid\left(\overline{\psi_{v}}\right) \overline{j_{v}}\right\rangle\left\langle j_{v}, \overline{j_{v}}\right|\right\}\left(j_{1} j_{2}\right) J_{v}\right\rangle
\end{aligned}
$$




$$
\begin{aligned}
& \times(-1)^{J_{N-3}+j_{s}+2 j_{v}+J_{\text {sub }}^{\prime}+J_{\text {sub }}+j_{v}^{\prime}+J_{\mathrm{tot}}} \\
& \times\left[J_{\text {sub }}, J_{G}, J_{\text {sub }}^{\prime}, J_{v}\right]^{1 / 2}\left\{\begin{array}{ccc}
J_{N-3} & j_{s} & J_{\text {sub }} \\
j_{v} & J_{\text {sub }}^{\prime} & J_{G}
\end{array}\right\} \\
& \times\left\{\begin{array}{ccc}
J_{\text {sub }} & j_{v} & J_{\text {sub }}^{\prime} \\
j_{v}^{\prime} & J_{\text {tot }} & J_{v}
\end{array}\right\}\left\langle\left(\psi_{2}^{\prime}\right) J_{G}|\mathbf{G}|\left(\psi_{s v}\right) J_{G}\right\rangle .
\end{aligned}
$$

\section{Example: Coulomb interaction between double-replacement configurations}

Finally we consider in slightly more detail the case of the interaction of two double-replacement symbolic states (30):

$$
\left\langle\Psi_{d}^{\prime}|\mathbf{G}| \Psi_{d}\right\rangle=\mathcal{M}_{v v}+\mathcal{M}_{s s}+\mathcal{M}_{s v} .
$$

The factorization of quantities independent of the virtual orbitals is seen clearly in the equations (34), (35) for $\mathcal{M}_{v v}$ and $\mathcal{M}_{s s}$, but the situation for $\mathcal{M}_{s v}$ given by equations (46), (47) is more complicated. To see this explicitly write (46)

$$
\mathcal{M}_{s v}=\sum_{j_{s}, j_{s}^{\prime}} \sum_{j_{v}, j_{v}^{\prime}} \sum_{J_{G}} \mathcal{C}_{\mathrm{sym}}\left\langle\left(j_{s}^{\prime}, j_{v}^{\prime}\right) J_{G}|\mathbf{G}|\left(j_{s}, j_{v}\right) J_{G}\right\rangle,
$$

with

$$
\begin{gathered}
\mathcal{C}_{\text {sym }}=\sum_{k} \mathcal{D}_{k} \mathcal{E}_{k}, \\
\mathcal{D}_{k}=2[k]\left[J_{v}, J_{v}^{\prime}\right]^{1 / 2} \Gamma_{k}\left(\Psi_{\text {sub }}^{\prime}, j_{s}^{\prime} ; \Psi_{\text {sub }}, j_{s}\right) \times \\
\times(-1)^{k+J_{\text {tot }}+J_{v}+J_{v}^{\prime}+J_{\text {sub }}-j_{s}+1 / 2}\left\{\begin{array}{ccc}
J_{v} & k & J_{v}^{\prime} \\
J_{\text {sub }}^{\prime} J_{\text {tot }} J_{\text {sub }}
\end{array}\right\} \\
\left.\left.\mathcal{E}_{k}=\delta_{\overline{j_{v}}, \overline{j_{v}^{\prime}}}\left(j_{v}, \overline{j_{v}} \mid\right\}\left(j_{1} j_{2}\right) J_{v}\right)\left(j_{v}^{\prime}, \overline{j_{v}^{\prime}} \mid\right\}\left(j_{1}^{\prime} j_{2}^{\prime}\right) J_{v}^{\prime}\right) \\
\times\left[J_{G}\right](-1)^{\overline{j_{v}}-1 / 2-J_{G}}\left\{\begin{array}{l}
J_{v} k J_{v}^{\prime} \\
j_{v}^{\prime} \overline{j_{v}} j_{v}
\end{array}\right\}\left\{\begin{array}{l}
j_{s} k j_{s}^{\prime} \\
j_{v}^{\prime} J_{G} j_{v}
\end{array}\right\}
\end{gathered}
$$

Now we see that the set of coefficients $\mathcal{E}_{k}$ must be computed in conjunction with the "radial" portion of the calculation for each choice of virtual orbitals $j_{1}, j_{2}, j_{1}^{\prime}, j_{2}^{\prime}$. But the set of coefficients $\mathcal{D}_{k}$ can be computed at the beginning as part of the "angular" segment of the problem. Especially note that the details of the (N-2)-body subconfigurations affect only the factors $\Gamma_{k}\left(\Psi_{\text {sub }}^{\prime}, j_{s}^{\prime} ; \Psi_{\text {sub }}, j_{s}\right)$ which are independent of the quantum numbers of the virtual orbitals, and can be computed and stored at the beginning.

To illustrate the use of these results, we consider the Coulomb interaction between selected double-replacement states in a simple symbolic expansion based on the ground configuration of neutral fluorine. If we begin by using a four-electron closed core $1 s^{2} 2 s^{2}$, we have the five-electron $J=3 / 2$ reference state

$$
\left|\Psi_{\text {ref }}\right\rangle=\left|\left\{\left(2 p_{-}{ }^{2}\right) 0,\left(2 p^{3}\right) 3 / 2\right\} 3 / 2\right\rangle,
$$

where we indicate the $j=l-1 / 2$ Dirac orbitals by the subscripted minus sign.

Now for a simple illustrative symbolic expansion we can define five subconfigurations:

$$
\begin{aligned}
& \Psi_{0}=\left[\left(2 p_{-}{ }^{2}\right) 0,(2 p) 3 / 2\right] 3 / 2, \\
& \Psi_{1}=\left[\left(2 p_{-}\right) 1 / 2,\left(2 p^{2}\right) 0\right] 1 / 2, \\
& \Psi_{2}=\left[\left(2 p_{-}\right) 1 / 2,\left(2 p^{2}\right) 2\right] 3 / 2, \\
& \Psi_{3}=\left[\left(2 p_{-}\right) 1 / 2,\left(2 p^{2}\right) 2\right] 5 / 2, \\
& \Psi_{4}=\left(2 p^{3}\right) 3 / 2 .
\end{aligned}
$$

These produce a total of 18 double-replacement symbolic states of form

$$
\left|\Psi_{d}\right\rangle=\left|\left\{\left(\Psi_{\text {sub }}\right) J_{\text {sub }},\left(n_{1} l_{1} j_{1}, n_{2} l_{2} j_{2}\right) J_{v}\right\} 3 / 2\right\rangle,
$$

namely

$\Psi_{0}: J_{\text {sub }}=3 / 2, \quad J_{v}=0,1,2,3, \quad 4$ symbolic states, $\Psi_{1}: J_{\text {sub }}=1 / 2, J_{v}=1,2, \quad 2$ symbolic states, $\Psi_{2}: J_{\mathrm{sub}}=3 / 2, J_{v}=0,1,2,3, \quad 4$ symbolic states, $\Psi_{3}: J_{\text {sub }}=5 / 2, J_{v}=1,2,3,4, \quad 4$ symbolic states, $\Psi_{4}: J_{\text {sub }}=3 / 2, J_{v}=0,1,2,3, \quad 4$ symbolic states.

The matrix of the Coulomb interaction $\mathbf{G}=\sum_{i<j} \frac{1}{r_{i j}}$ is divided into $18 \times 18$ blocks, each block connecting a pair of symbolic states. For each of these blocks, we can calculate the coefficients $\mathcal{D}_{k}$ at the beginning of the project.

For example for the particular block

$\left\langle\Psi_{d}^{\prime}|\mathbf{G}| \Psi_{d}\right\rangle=$

$\left\langle\left\{\left(\Psi_{2}\right) 3 / 2,\left(j_{1}^{\prime} j_{2}^{\prime}\right) 2\right\} 3 / 2|\mathbf{G}|\left\{\left(\Psi_{3}\right) 5 / 2,\left(j_{1} j_{2}\right) 2\right\} 3 / 2\right\rangle$, consider the matrix element of form $\mathcal{M}_{s v}$ (30). We find from (44) the results

$$
\begin{aligned}
& \Gamma_{k}\left(\Psi_{2}, 2 p_{-} ; \Psi_{3}, 2 p_{-}\right)=-\sqrt{8 / 5} \delta_{k 1}, \\
& \Gamma_{k}\left(\Psi_{2}, 2 p_{-} ; \Psi_{3}, 2 p\right)=0, \\
& \Gamma_{k}\left(\Psi_{2}, 2 p \quad ; \Psi_{3}, 2 p_{-}\right)=0, \\
& \Gamma_{k}\left(\Psi_{2}, 2 p \quad ; \Psi_{3}, 2 p\right)=(2 / 5) \delta_{k 1}-(2 \sqrt{6} / 5) \delta_{k 3} .
\end{aligned}
$$


Now we use (66) to get the required values of $\mathcal{D}_{k}$ :

Case $\left(\Psi_{2}, 2 p_{-} ; \Psi_{3}, 2 p_{-}\right): \mathcal{D}_{k}=2 \sqrt{21 / 5} \delta_{k 1}$,

Case $\left(\Psi_{2}, 2 p ; \Psi_{3}, 2 p\right): \mathcal{D}_{k}=\sqrt{42} / 5\left(\delta_{k 1}-4 \delta_{k 3}\right)$.

Note that these $\mathcal{D}_{k}$ factors are independent of the $(n, l, j)$ quantum numbers of all four virtual orbitals. Thus for this block we have

$$
\begin{aligned}
\mathcal{M}_{s v}= & 2 \sqrt{\frac{21}{5}} \sum_{v^{\prime}, v, J_{G}} \mathcal{E}_{1}\left(j_{s}^{\prime}=j_{s}=2 p_{-}\right) \\
& \times\left\langle\left(2 p_{-}, v^{\prime}\right) J_{G}|\mathbf{G}|\left(2 p_{-}, v\right) J_{G}\right\rangle \\
+ & \frac{1}{5} \sqrt{42} \sum_{v^{\prime}, v, J_{G}} \mathcal{E}_{1}\left(j_{s}^{\prime}=j_{s}=2 p\right) \\
& \times\left\langle\left(2 p, v^{\prime}\right) J_{G}|\mathbf{G}|(2 p, v) J_{G}\right\rangle \\
- & \frac{4}{5} \sqrt{42} \sum_{v^{\prime}, v, J_{G}} \mathcal{E}_{3}\left(j_{s}^{\prime}=j_{s}=2 p\right) \\
& \times\left\langle\left(2 p, v^{\prime}\right) J_{G}|\mathbf{G}|(2 p, v) J_{G}\right\rangle .
\end{aligned}
$$

In these summations the label $v$ takes on just 2 values: the two orbitals $\left(n_{1}, l_{1}, j_{1}\right)$ and $\left(n_{2}, l_{2}, j_{2}\right)$; similarly for $v^{\prime}$. The quantum number $J_{G}$ takes on all values allowed by the angular momentum coupling of an electron from the subconfiguration with an electron in one of the virtual orbitals. The coefficients $\mathcal{E}_{k}$ in (73) do depend on the virtual orbitals, but are given explicitly by equation (66). Note that the 2-body MCFP factors in that equation are simply 0 or 1 if the electrons are equivalent, and $\pm \sqrt{1 / 2}$ if they are inequivalent.

\section{Summary}

Within the framework of the symbolic expansion, matrix elements of two-body operators can be expressed as a sum of terms, where each term is a product of three factors:

- An angular quantity independent of the virtual orbitals.

- An angular quantity dependent on the virtual orbitals.

- A two-electron matrix element.

Note that the first two quantities involve no radial functions; the third includes both angular and radial integrals, but is readily computed by standard methods since it involves only two electrons.
We have considered a symbolic expansion containing three types of state functions: reference states, single-replacement states, and double-replacement states. Thus there are six types of matrix elements for a two-body operator, and we have derived equations for the angular integrals for each type separately. These equations $(21,27,30,34,35,46-51,62,63)$ constitute our principal results. In each case the separation into the three factors listed above is shown explicitly.

These results have been derived [13] under the assumption that the virtual (or correlation) orbitals were distinct from the occupied orbitals. Otherwise the angular quantum numbers of the virtual orbitals were not specified. The extent to which this separation of occupied and virtual orbitals is necessary requires further investigation. Clearly the subconfigurations consist only of occupied orbitals.

\section{References}

[1] C. Froese Fischer and D. Ellis, Lithuanian J. Phys. 44, 121-134 (2004).

[2] A.P. Jucys and A.A. Bandzaitis, The Theory of Angular Momentum in Quantum Mechanics, 2nd ed. (Mokslas, Vilnius, 1977) [in Russian].

[3] S. Meshkov, Theory of complex spectra, Phys. Rev. 91, 871-876 (1953).

[4] L. Armstrong Jr., Matrix elements between configurations having several open shells. II, Phys. Rev. 172, 1823 (1968).

[5] A. Starace and L. Armstrong Jr., Photoionization cross sections for atomic chlorine using an open-shell random-phase approximation, Phys. Rev. A 13, 18501865 (1976).

[6] G. Merkelis, Jucys graphs of angular momentum theory, Lithuanian J. Phys. 44, 91-120 (2004).

[7] D.A. Varshalovich, A.N. Moskalev, and V.K. Khersonskii, Quantum Theory of Angular Momentum (World Scientific, Singapore, 1988).

[8] J.S. Briggs, Evaluation of matrix elements from a graphical representation of the angular integral, Rev. Mod. Phys. 43, 189-230 (1971).

[9] K.-N. Huang, Graphical evaluation of relativistic matrix elements, Rev. Mod. Phys. 51, 215-236 (1979).

[10] A.P. Jucys, I.B. Levinson, and V.V. Vanagas, Mathematical Apparatus of the Theory of Angular Momentum (Israel Program for Scientific Translations, Jerusalem, 1962).

[11] Z. Rudzikas, Theoretical Atomic Spectroscopy (Cambridge University Press, Cambridge, UK, 1997).

[12] M. Rotenberg, R. Bivins, N. Metropolis, and J.K. Wooten Jr, The 3-j and 6-j Symbols (Technology Press, M.I.T., Cambridge, MA, 1959). 
[13] R. Matulioniene, Angular Momentum Algebra for Symbolic Expansions in Atomic Structure Theory, $\mathrm{PhD}$ thesis, University of Toledo, Ohio, USA (1999).
[14] R.D. Cowan, The Theory of Atomic Structure and Spectra (University of California Press, Berkeley, 1981).

\title{
SIMBOLINIŲ BŪSENŲ SĄVEIKA ATOMINĖS SANDAROS SKAIČIAVIMUOSE
}

\author{
R. Matulioniené ${ }^{\mathrm{a}}$, D. Ellis ${ }^{\mathrm{a}}$, C. Froese Fischer ${ }^{\mathrm{b}}$ \\ ${ }^{\text {a }}$ Toledo universitetas, Toledas, Ohajas, JAV \\ ${ }^{\mathrm{b}}$ Vanderbilto universitetas, Nešvilis, Tenesis, JAV
}

\section{Santrauka}

Išvestos bendros lygtys kampiniams koeficientams, kurie reikalingi atliekant atominès sandaros skaičiavimus, panaudojant skleidinius simbolinėmis būsenomis. Šiuo naujuoju būdu energija išreiškiama ne kinetinès energijos ir Sleterio integralais, bet dvielektroniais matriciniais elementais, kurių koeficientai nepriklauso nuo vienelektronių kvantinių skaičių, esančių tuose matriciniuose elementuose. Pateikti konkretūs rezultatai simetrinio skaliarinio dvidalelio operatoriaus su viengubo ir dvigubo keitimo simbolinėmis būsenomis matriciniams elementams. Išvedimui naudotas jj ryšys, nelygiaverčių elektronų kilminiai koeficientai ir judejimo kiekio momento diagraminė algebra. 